\title{
Highlights from the 2017 American Heart Association Scientific Sessions in Anaheim, California
}

\author{
Dylan L. Steen ${ }^{1}$
}

Published online: 7 March 2018

๑) Springer Science+Business Media, LLC, part of Springer Nature 2018

The Journal of Thrombosis and Thrombolysis is proud to present the first article in a new section entitled "National and International Meeting Highlights". Each article in this series will review some of the major studies or guideline updates presented at international conferences. The conferences covered in this series will include the American Heart Association Annual Scientific Sessions, American Society of Hematology Annual Meeting, American College of Cardiology Annual Scientific Session, International Society of Thrombosis and Haemostasis Biennial Congress, and European Society of Cardiology Annual Congress. Although all the exciting presentations at each conference cannot be covered in these articles, we hope this series provides readers with a taste of the ones that may shape guidelines and practice.

\section{New insights from CANTOS}

The primary results of the Canakinumab Antiinflammatory Thrombosis Outcomes Study (CANTOS) were first presented at the 2017 ESC Congress [1]. This ground-breaking trial tested the "pure" anti-inflammatory drug (i.e. no impact on lipids), canakinumab, a monoclonal antibody targeting interleukin-1 $\beta$, in the reduction of recurrent major adverse cardiovascular events (MACE). The investigators found a $15 \%$ relative risk reduction with canakinumab in MACE, providing validation of the "inflammatory hypothesis", targeting of inflammation in atherosclerotic cardiovascular disease (ASCVD) reduction, and spurring excitement for further investment in understanding anti-inflammatory mechanisms and related drug discovery.

At the AHA, Dr. Ridker presented subanalyses of CANTOS that demonstrated that the benefit of canakinumab did

Dylan L. Steen

dylan.steen@uc.edu

1 Cincinnati, USA not differ in subgroups of varying baseline clinical characteristics, including the LDL-C [2]. In addition, analyses also demonstrated that on-treatment high-sensitivity C-reactive protein (hsCRP), a biomarker of inflammation, could identify responders to canakinumab (Table 1). These data demonstrated that the benefits of canakinumab were directly related to the magnitude of hsCRP reduction and that this biomarker can serve as a valuable target to assess treatment response.

\section{PRESERVE trial primary results}

The use of contrast material in cardiovascular angiography carries with it the well-known risk of acute kidney injury and long term renal complications. Investigators have long hypothesized that the risk of renal complications might be reduced by sodium bicarbonate infusion or oral acetylcysteine, yet previous studies have yielded inconclusive results. The Prevention of Serious Adverse Events Following Angiography (PRESERVE) trial, a $2 \times 2$ factorial design trial, randomized subjects to sodium bicarbonate infusion versus sodium chloride infusion and acetylcysteine versus placebo [3]. The primary endpoint was death, need for dialysis, or a persistent increase of at least $50 \%$ from baseline in serum creatinine at 90-104 days after angiography. The study which intended to enroll 7680 patients was stopped after 5177 patients were randomized (4993 included in the primary analysis group) after a preplanned interim analysis demonstrated no between-group differences. The results of the study provided convincing evidence that there is neither incremental benefit of sodium bicarbonate versus sodium chloride infusion nor of acetylcysteine administration. 
Table 1 Adjusted hazard ratios for prespecified endpoints according to on-treatment hsCRP

\begin{tabular}{llll}
\hline Outcome & Placebo & hsCRP $\geq 2 \mathrm{mg} / \mathrm{dL}$ & hsCRP $<2 \mathrm{mg} / \mathrm{dL}$ \\
\hline MI, stroke or all-cause mortality & 1 (ref) & $0.93(0.83-1.05)$ & $0.73(0.65-0.82)$ \\
Cardiovascular death & 1 (ref) & $0.99(0.82-1.21)$ & $0.69(0.56-0.85)$ \\
All-cause mortality & 1 (ref) & $1.05(0.90-1.22)$ & $0.69(0.58-0.81)$ \\
\hline
\end{tabular}

Data are reported as adjusted hazard ratios with $95 \%$ confidence intervals

\section{TRISC III primary results}

Strategies of liberal versus restrictive thresholds for packed red blood cell (PRBC) transfusion have undergone evaluation across a variety of conditions. The unknown balance of risks of anemia-induced tissue hypoxia versus risks due to transfusions, led the investigators of the Transfusion Requirements in Cardiac Surgery (TRISC) III trial to evaluate these strategies in adult patients undergoing cardiac surgery with cardiopulmonary bypass [4]. Using a noninferiority design, patients with a EuroSCORE I of 6 or higher (higher scores indicating higher risk of death after cardiac surgery) were randomized 1:1 to a transfusion threshold of: (1) $7.5 \mathrm{~g} / \mathrm{dL}$ intraoperatively and postoperatively, versus (2) $9.5 \mathrm{~g} / \mathrm{dL}$ intraoperatively and in the ICU and then $8.5 \mathrm{~g} / \mathrm{dL}$ in the non-ICU ward. The primary endpoint was a composite of death, nonfatal myocardial infarction, stroke, or new-onset renal failure.

At baseline, mean hemoglobin concentration was $13.1 \mathrm{~g} /$ dL. During the post-operative period, the hemoglobin concentration between the groups differed by approximately $1 \mathrm{~g} / \mathrm{dL}$. In the restrictive-threshold group $52.3 \%$ received a transfusion versus $72.6 \%$ the liberal-threshold group. The primary endpoint occurred in 11.4 versus $12.5 \%$ in the restrictive versus liberal-threshold groups (Table 2). These data demonstrate that a more restrictive approach to transfusion is noninferior to a more liberal approach for patients undergoing cardiac surgery with cardiopulmonary bypass.

\section{New Insights from RE-DUAL PCI}

The primary results of the Randomized Evaluation of Dual Antithrombotic Therapy with Dabigatran versus Triple Therapy with Warfarin in patients with Nonvalvular Atrial
Fibrillation Undergoing Percutaneous Coronary Intervention (REDUAL-PCI) study were first presented at the 2017 ESC Congress [5]. This noninferiority trial randomized patients with nonvalvular atrial fibrillation undergoing PCI to either dabigatran $110 \mathrm{mg}$ BID plus a P2Y12 inhibitor, dabigatran $150 \mathrm{mg}$ BID plus a P2Y12 inhibitor, or warfarin plus both a P2Y12 inhibitor and aspirin. As expected, the primary safety end point of first major or clinically relevant nonmajor ISTH-defined bleeding was substantially reduced in the $110 \mathrm{mg}$ dabigatran arm compared to the warfarin tripletherapy comparator group, 15.4 versus $26.9 \%$, respectively, and in the $150 \mathrm{mg}$ dabigatran arm compared to its warfarin triple-therapy comparator group, 20.2 versus $25.7 \%$, respectively. Of note, the composite efficacy endpoint of thromboembolic events, death, and unplanned revascularization did not differ between the combined dabigatran arms versus the warfarin triple-therapy arm, 13.7 versus $13.4 \%$.

At the AHA, Dr. Oldgren presented subgroup analyses for key baseline clinical subgroups, including stenting for ACS versus non-ACS indication, drug-eluting versus baremetal stent use, and P2Y12 choice of clopidogrel versus ticagrelor. The investigators found no signification interactions by subgroups and concluded that the main study results were consistent amongst them. These findings support use of non-warfarin based strategies in these patients as well as the safety of ticagrelor if desired in those at higher thrombotic risk.

\section{Release of 2017 hypertension guidelines}

Finally, the new ACC/AHA hypertension guidelines were released [6]. Although this article doesn't permit coverage of this long and comprehensive document, some key points are worth mentioning:
Table 2 Outcomes in the perprotocol population

\begin{tabular}{lccc}
\hline Outcome & Restrictive threshold & Liberal threshold & Odds ratio (95\% CI) \\
\hline Primary endpoint (\%) & 11.4 & 12.5 & $0.90(0.76-1.07)$ \\
Death (\%) & 3.0 & 3.6 & $0.85(0.62-1.16)$ \\
Stroke (\%) & 1.9 & 2.0 & $0.92(0.61-1.38)$ \\
Myocardial infarction (\%) & 5.9 & 5.9 & $1.00(0.79-1.27)$ \\
Renal failure with dialysis (\%) & 2.5 & 3.0 & $0.84(0.60-1.19)$ \\
\hline
\end{tabular}


(1) The definitions of systolic and diastolic blood pressure (SBP and DBP) categories have been modified. Normal $\mathrm{BP}$ is $<120 /<80 \mathrm{~mm} \mathrm{Hg}$; elevated BP is $120-129 /<80$; hypertension stage 1 is $130-139$ or $80-89$; and hypertension stage 2 is $\geq 140$ or $\geq 90$.

(2) BP should be estimated using $\geq 2$ readings on $\geq 2$ occasions. Out-of-office and self-monitoring are recommended to confirm the diagnosis and titrate therapy.

(3) Use of BP-lowering medication is recommended in patients with cardiovascular disease with an average $\mathrm{SBP} \geq 130$ or $\mathrm{DBP} \geq 80$. Medication is also recommended in primary prevention for those with a 10-yearr ASCVD risk $\geq 10 \%$ with the same BP measurements. In primary prevention, those with a 10 -year risk $<10 \%$ and $\mathrm{SBP} \geq 140$ or DBP $\geq 90$ should also be treated.

(4) First-line therapy for stage 1 hypertension includes thiazide diuretics, calcium channel blockers, and angiotensin-converting-enzyme inhibitors or angiotensin II receptor blockers. Two first-line drugs of different classes are recommended for stage 2 hypertension. Note that for specific conditions (e.g. heart failure with reduced ejection fraction, thoracic aortic disease), BP medication recommendations will vary (e.g. betablockers).

\section{References}

1. Ridker PM, Thuren ET, MacFadyen JG et al (2017) Antiinflammatory therapy with canakinumab for atherosclerotic disease. NEJM 21(12):1119-1131 377)

2. Ridker PM, MacFadyen JG, Everett BM et al (2017) Relationship of C-reactive protein reduction to cardiovascular event reduction following treatment with canakinumab: a secondary analysis from the CANTOS randomized trial. Lancet. https://doi.org/10.1016/ S0140-6736(17)32814-3

3. Weisbord SD, Gallagher M, Jneid H et al (2017) Outcomes after angiography with sodium bicarbonate and acetylcysteine. NEJM. https://doi.org/10.1056/NEJMoa1710933

4. Mazer CD, Whitlock RP, Fergusson DA et al (2017) Restrictive or liberal red-cell transfusion for cardiac surgery. NEJM 30(22):2133-2144 377)

5. Cannon CP, Bhatt DL, Oldgren J et al (2017) Dual antithrombotic therapy with dabigatran after PCI in atrial fibrillation. NEJM 19(16):1513-1524 377)

6. Whelton PK, Carey RM, Aronow WS et al (2017) 2017 ACC/ AHA/AAPA/ABC/ACPM/AGS/AphA/ASH/ASPC/NMA/PCNA guideline for the prevention, detection, evaluation, and management of high blood pressure in adults: a report of the American College of Cardiology/American Heart Association Task Force on Clinical Practice Guidelines. JACC. https://doi.org/10.1016/j. jacc.2017.11.006 